\title{
Erratum to: Groundwater quality assessment using data clustering based on hybrid Bayesian networks
}

Pedro A. Aguilera • Antonio Fernández •

Rosa F. Ropero · Luís Molina

Published online: 12 April 2013

(C) Springer-Verlag Berlin Heidelberg 2013

Erratum to: Stoch Environ Res Risk Assess (2013)

\section{7:435-447}

DOI 10.1007/s00477-012-0676-8

Unfortunately mistakes were introduced in two equations in this article. Please find below the corrected equations.

$f\left(y_{1}, y_{2}\right)= \begin{cases}2+\mathrm{e}^{3 y_{1}+y_{2}}+\mathrm{e}^{y_{1}+y_{2}} & \text { if } 0 \leq y_{1} \leq 1,0 \leq y_{2} \leq 2 \\ 1+\mathrm{e}^{y_{1}+y_{2}} & \text { if } 0 \leq y_{1} \leq 1,2 \leq y_{2} \leq 3 \\ \frac{1}{4}+\mathrm{e}^{2 y_{1}+y_{2}} & \text { if } 1 \leq y_{1} \leq 2,0 \leq y_{2} \leq 2 \\ \frac{1}{2}+5 \mathrm{e}^{y_{1}+y_{2}} & \text { if } 1 \leq y_{1} \leq 2,2 \leq y_{2} \leq 3\end{cases}$

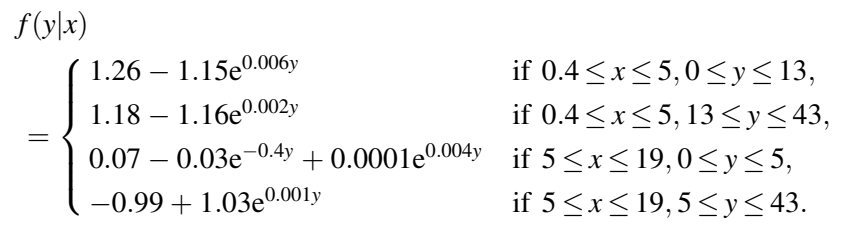

$f(y \mid x)$

$=\left\{\begin{array}{l}1.26-1.15 \mathrm{e}^{0.006}-1.16 \mathrm{e}^{0.002 y} \\ 0.07-0.03 \mathrm{e}^{-0.4 y}+0.0001 \mathrm{e}^{0.004 y} \\ -0.99+1.03 \mathrm{e}^{0.001 y}\end{array}\right.$

if $0.4 \leq x \leq 5,13 \leq y \leq 43$,

if $5 \leq x \leq 19,0 \leq y \leq 5$
if $5 \leq y \leq 43$.

The online version of the original article can be found under doi:10.1007/s00477-012-0676-8.

P. A. Aguilera - R. F. Ropero

Informatics and Environment Laboratory, Department of Plant

Biology and Ecology, University of Almería, Almería, Spain

e-mail: aguilera@ual.es

R. F. Ropero

e-mail: rfr723@alboran.ual.es

A. Fernández $(\bowtie)$

Department of Statistics and Applied Mathematics, University

of Almería, Almería, Spain

e-mail: afalvarez@ual.es

L. Molina

Department of Hydrogeology and Analytic Chemistry,

University of Almería, Almería, Spain

e-mail: 1molina@ual.es 